\title{
How Effective and Humane Is Trap-Neuter-Release (TNR) for Feral Cats?'
}

\author{
Mark Hostetler, Samantha M. Wisely, Steve Johnson, Elizabeth F. Pienaar, and Martin Main²
}

\section{Introduction}

The number of feral, abandoned cats in the United States is estimated to be between 30 and 80 million individuals (Loss et al. 2018; Jessup 2004). As the number of feral cats continues to increase, land managers, public health officials, and private citizens are voicing concerns about how to address the nuisance and public health impacts, as well as animal welfare concerns, that feral cats create. Feral cat management falls into two main categories of control techniques: lethal and nonlethal. Gaining popularity in the United States, the trap-neuter-release (TNR) strategy for feral cat management proposes to reduce feral cat populations without euthanasia. Typically, cats are live-trapped and taken to a nearby veterinarian, where the cats are neutered/spayed, sometimes vaccinated for rabies, and then released back to the area where they were trapped (Figure 1 ). Because the sterilized cats can no longer reproduce, the assumption is that over time the numbers of feral cats in a colony will decline, and eventually the colony will cease to exist.

In Florida, the TNR strategy is being considered by many county governments as an option to manage/control feral cat colonies. Brevard, Palm Beach, Volusia, Gilchrist, and Okaloosa counties in Florida have amended their ordinances to make TNR legal (Hatley 2019). But is TNR an effective and humane method to reduce feral cat populations? Below, we discuss the pros and cons of creating
TNR cat colonies. We investigate peer-reviewed, scientific literature to determine if TNR is an effective way to reduce feral cat colonies and whether the method is humane.

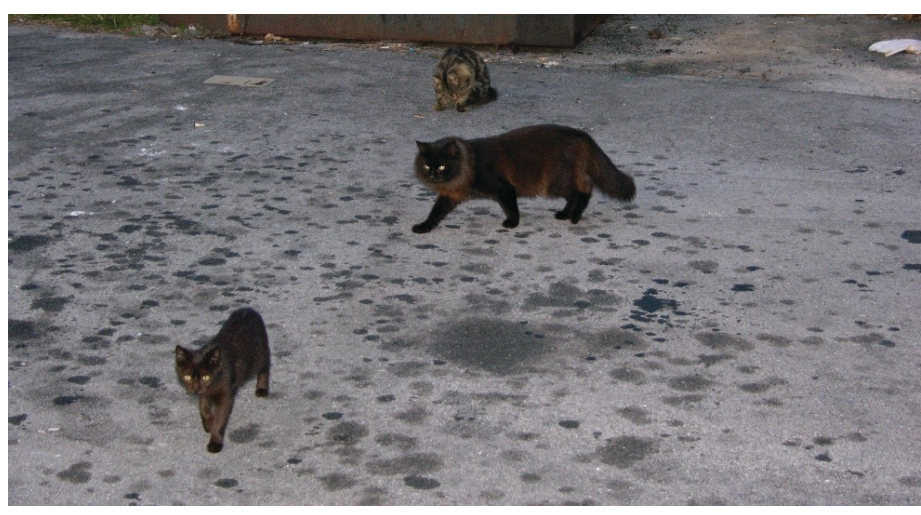

Figure 1. An example of a feral cat colony.

Credits: Steve Johnson, UF/IFAS

\section{Does TNR reduce cat populations over time?}

\section{Studies That Claim That TNR Does Not Reduce Cat Populations}

One of the goals of TNR programs is to reduce feral cat populations in an area. The idea is that neutering/spaying cats and returning them to the environment will prevent them from reproducing. Over time, the number of feral cats in an area will decrease, and eventually they will disappear. The first study we reviewed was published in the Natural Areas Journal (Castillo and Clarke 2003). Here, researchers

1. This document is WEC423, one of a series of the Department of Wildlife Ecology and Conservation, UF/IFAS Extension. Original publication date March 2020. Visit the EDIS website at https://edis.ifas.ufl.edu for the currently supported version of this publication.

2. Mark Hostetler, professor; Samantha M. Wisely, professor; Steve Johnson, associate professor; Elizabeth F. Pienaar, associate professor; and Martin Main, professor; Department of Wildlife Ecology and Conservation, UF/IFAS Extension, Gainesville, FL 32611.

The Institute of Food and Agricultural Sciences (IFAS) is an Equal Opportunity Institution authorized to provide research, educational information and other services

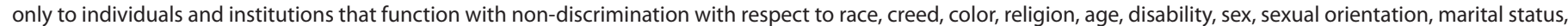

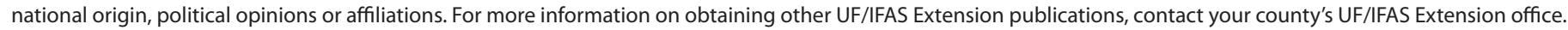
U.S. Department of Agriculture, UF/IFAS Extension Service, University of Florida, IFAS, Florida A \& M University Cooperative Extension Program, and Boards of County Commissioners Cooperating. Nick T. Place, dean for UF/IFAS Extension. 
tracked two TNR colonies in two public parks in MiamiDade County, Florida. Original populations were 25 cats at one park and 56 cats at another. Cats were monitored from 1999 to 2001 using a photographic capture/recapture technique. In both parks, managed cat populations increased over time due primarily to the introduction of new cats to these colonies, either through illegal dumping or as the result of stray cats being attracted to the colonies (an increase of 27 cats in one park and 61 cats in the other). A second study we reviewed used San Diego County, California, and Alachua County, Florida, TNR data and found that TNR did not reduce the feral cat population growth rate or the proportion of pregnant feral cats (Foley et al. 2005). Here, they applied a population growth model and found that in order for a TNR colony to decline over time, $71 \%$ to $94 \%$ of the cats needed to be neutered. These percentages of neutering were higher than what occurred in the field, and thus the models indicated that populations would increase. Both of these studies used data collection methods for estimating abundance that are scientifically accepted. Overall, these studies reached the conclusion that TNR did not significantly reduce cat population sizes, but that population sizes increased due to immigration of other cats and the difficulty of trapping all cats and neutering them. Immigration is the movement of cats from the surrounding area into the target population.

\section{Studies That Claim That TNR Does Reduce Cat Populations}

We first reviewed a study published in the Journal of the American Veterinary Medical Association (Centonze and Levy 2002). This study was a survey of caretakers of the TNR cat colonies in north central Florida. The authors asked caretakers to report the initial size of the colony and to estimate the size of the colony after approximately 8 months. This study was conducted across 132 colonies that initially totaled 920 cats. The authors reported that the total number of cats declined from 920 to 678 (a 27\% reduction). They reported 151 deaths, 149 disappearances, 238 adoptions, 498 births and 103 immigrants. However, taking into account all of the aforementioned parameters, the final cat population should be calculated as 983 , not 678 . The numbers generated in the publication do not add up because, as the authors stated, the estimated cat populations were "...based on the recollections of individual caretakers," and the population fluctuated as the cats that belonged to the colonies changed. Consequently, the reliability of the estimated cat numbers is questionable (i.e., human error in estimating the number of cats); it was not based on a scientifically accepted way to estimate abundance. In addition, most of the cat colonies included in the study were very small (an average of 7 cats per colony), with the largest colony being 89 cats. The small colony sizes in the study limit how applicable this study is to other larger TNR colonies in the United States. Because of the disparity between the number of cats reported and the population trend (the number of reported cats was higher at the end of the study, yet observers reported a decrease in the colony size), we do not consider the methods used in this study to be reliable. Furthermore, adoptions accounted for $26 \%$ of the original cat population, an outcome that was needed to somewhat offset immigration and births.

In a review study by Crawford, Calver, and Fleming (2019), authors noted that there were very few studies that tracked a TNR cat colony from initial population size to final population size. They only found 11 studies that did this and found that, typically, cat colonies were tracked for less than 3 years. Ten of these studies were in urban areas and one in a rural setting. From these studies, the number of cats that were adopted was reported in 7 colonies. Of these, 5 colonies decreased (ranging from a $1 \%$ to a $72 \%$ reduction), and 2 colonies increased (ranging from a $25 \%$ to a $175 \%$ increase). For the 5 colonies that decreased, adoption rates ranged from $18 \%-80 \%$. Adoption rates were calculated by dividing the number of reported adopted cats by the number of cats in the initial colony added to the number of new cats that joined the colony. They noted that in each study, new cats joined the colonies, indicating that TNR colonies are not closed colonies and have immigration occurring due to abandoned cats or cats attracted to the colonies. Most studies concluded that TNR cat colonies were prone to attracting abandoned and stray cats (Longcore et al. 2009).

Another study had volunteers monitor TNR cat colonies at the University of Central Florida (Levy et al. 2003). Here, 68 cats from 11 colonies in 1996 were reduced to 23 cats in 2002. These colonies attracted additional cats, but overall numbers decreased due to deaths, euthanasia (of sick cats) and a high rate of adoption (47\%). This adoption rate was similar to the removal rate $(50 \%)$ calculated from a population model of TNR colonies (Andersen et al. 2004), which is necessary to reduce feral cat populations. For the TNR colonies at the University of Central Florida, the high adoption rate was the primary reason for the reduction in TNR cat populations.

Other case studies that found a reduction in cat colonies through TNR also had high adoption/removal rates: 39\% adopted, euthanized, or relocated in Chicago (Spehar and Wolf 2018); 43\% estimated adopted or euthanized in Newburyport, MA (Spehar and Wolf 2017); and 32\% 
adopted or euthanized in Sydney, Australia (Swarbrick and Rand 2018). Each of these studies found a reduction in cat colonies, largely owing to adoption and removal. There is less evidence that the reduction in cat colonies was attributable solely to spaying/neutering of cats, and a concerted effort was needed to remove cats to help decrease populations over time.

Many of the above studies (e.g., Centonze and Levy 2002; Levy et al. 2003) were conducted with volunteers and used surveys of caretakers or veterinary clinic records to estimate abundance. These studies did not use standardized field observations; thus, results are likely not accurate. To highlight the discrepancy in data collection, we present data from a study where only one caretaker surveyed cats at various feeding stations in Key Largo from 1999 to 2013 (Kreisler, Cornell, and Levy 2019). This caretaker did not report how often each feeding station was sampled. The number of feeders changed from year to year, and many of the feeding stations were located near each other, so that cats very likely ate from multiple stations, yet the caretaker provided no rationale for how double counting was avoided. Furthermore, the report did not state what happened to the cats in each of the colonies: were they adopted, killed, or euthanized? The report did not provide this information.

In a large study of 72,970 TNR cats across six cities (Spehar and Wolf 2019), researchers measured feline intake to municipal shelters over a three-year period. They found a median reduction of $32 \%$ in feline intake and a median decline of $83 \%$ in shelter euthanasia. Although fewer cats were taken to shelters, it is not clear that cat colonies decreased in size. The authors attempted to compare the number of DOA (dead on arrival) cats before and after implementing the TNR program, but inconsistencies in the data meant that the authors could not demonstrate a consistent decline in cat colonies. Other studies have reported similar results where TNR along with targeted adoption reduced the number of cats taken into shelters, but overall reductions of cat populations across the cities examined in these studies were not measured (Levy, Isaza, and Scott 2019).

Another field study in Rome, Italy, found that TNR programs did reduce population size (16\% to $32 \%$ ) (Natoli et al. 2006). Researchers surveyed 103 cat colonies from 1991 to 2000 and found that some of the colonies increased and others decreased. The authors found that cat immigration from nearby neighborhoods was around 21 percent, and that the immigration was due to abandoned cats from nearby neighborhoods. They concluded that TNR programs alone may not decrease cat populations unless nearby residents are educated to neuter their own cats and not to abandon cats.

Finally, a study that modeled various cat-management practices reported that the best practice for control was TNR (Boone et al. 2019). This study modeled population fluctuation using estimated population parameters with different hypothetical management strategies, such as TNR at low and high removal intensities. At the end of 10 years using these models, outputs measured were population size and preventable deaths. Preventable deaths were defined as the number of cats that would not die as the result of a particular management strategy. The study reported that high-intensity TNR ( $50 \%$ of cats sterilized every 6 months) was the best solution for minimizing preventable deaths. However, results indicated that the best strategy to reduce cat populations was $50 \%$ removal of cats, either through euthanasia or adoption. One shortcoming of the modeling effort was the use of an immigration rate of $2 \%$. Other studies report a substantially higher rate of immigration (e.g., Crawford, Calver, and Fleming 2019). Using values greater than $2 \%$ would have greatly increased the population size over time. The authors state this was an arbitrary number. Thus, they should have tried the scenarios at different immigration rates. Another shortcoming of the model was the assumption that a given environment's carrying capacity for a cat colony is equal to the established cat colony's population size. An environment's carrying capacity for a given species of animal is the maximum number of animals that can subsist on the resources in the environment (food, shelter, etc.). Because TNR programs typically feed cats, the carrying capacity would likely increase with the establishment of a TNR plan, because the extra food would mean that the environment could support additional cats. Reliable food sources can cause cats to become more social and less territorial (Levy and Crawford 2004), resulting in more cats in a given area (Schmidt et al. 2007).

Overall, it appears that TNR cat colonies were reduced only if there were high rates of adoption/removal of cats (around $50 \%$ or more), if there were high rates of neutered cats, and if there were low rates of immigration by cats. Without these, the colonies would not decline in size over time.

\section{How humane are TNR programs for the cats?}

Regardless of whether TNR programs are effective in reducing feral cat populations, it is important to assess whether these programs are humane. Although some people feel that TNR programs are the most humane 
method of managing feral cat populations (e.g., https:// www.neighborhoodcats.org/how-to-tnr/getting-started/ what-is-tnr), animal rights organizations do not necessarily agree. PETA (People for the Ethical Treatment of Animals) states, "Having witnessed the painful deaths of countless feral cats, we cannot in good conscience advocate trapping, altering, and releasing (e.g., TNR) as a humane way to deal with overpopulation and homelessness" (https://www.peta. org/about-peta/why-peta/feral-cats/; accessed 1/7/20).

Outdoor cats, whether they are on their own, in unmanaged colonies, or in TNR colonies, are subject to a variety of injuries, trauma, poisoning, and death. They scavenge dead animals, eat refuse out of garbage cans, and drink dirty water from parking lots (Crawford et al. 2019). Many free-ranging cats are brought into veterinary clinics because they have ingested toxic compounds (Crawford et al. 2019). Trauma, especially from being hit by cars or injured/killed by dogs or coyotes, is a serious risk for outdoor cats. In one study, $18 \%$ of 164 cats in TNR colonies were killed by vehicles (Nutter 2006). Even human cruelty is a factor when people shoot cats or purposely injure them by other means (e.g., Vnuk et al. 2016).

In a TNR study in Key Largo, Florida, of captured cats brought into a vet clinic (total = 2,530), $441(17 \%)$ were euthanized for being unhealthy or retrovirus-positive, and 209 (8\%) were dead on arrival through high trauma (e.g., hit by cars) or other unknown reasons (Kreisler, Cornell, and Levy 2019). Although 1,111 cats were returned to the colonies after veterinary treatment, Kreisler, Cornell, and Levy (2019) stated that "return [of cats to TNR colonies] rather than routine euthanasia of trapped cats [may] increase animal suffering due to non-retroviral disease or trauma (in other words, that free-roaming cats would be better off dead)." The average age of cats in this study was 82.1 months when they were euthanized and the average age of DOA/MIA cats was 58.7 months, indicating that many of these outdoor cats ended up being very sick and/ or killed by the time they were 7 years old. In one study in Raleigh, North Carolina, 127 of 169 (75\%) kittens died or disappeared in free-roaming managed colonies (Nutter et al. 2004). One study estimated that free-ranging domestic cats on farmsteads survived on average 3-5 years, and less than $1 \%$ survived 7 years or more (Warner 1985). Although few concrete studies have been published, various humane societies and veterinarian organizations estimate that indoor cats live longer on average than outdoor cats (e.g., American Veterinary Medicine Association, https://www. avma.org/policies/free-roaming-owned-cats).
Cats in TNR colonies may have high parasite loads, carry diseases that spread to other cats, compromise the health and welfare of wild and domestic animals, and threaten human safety (Wilson et al. 1994; Crawford et al. 2019). In New South Wales, Australia, 79\% of feral cats had feline immunodeficiency virus (FIV), 64\% had gingivitis, 54\% had throat conditions, and $23 \%$ had cat flu (Wilson et al. 1994). Feral cats have fleas, ticks, and lice and are susceptible to the diseases that these ectoparasites carry (Mohd et al. 2013; Lefkaditis et al. 2015). Gastrointestinal parasites, including roundworms and tapeworms (Waap et al. 2014), are also fairly common in feral and outdoor domestic cats (Chalkowski et al. 2019).

Treatment for the above diseases and parasites can be costly, and in many situations the cats in TNR colonies need to be recaptured for follow-up treatment. Recapturing feral cats can be very difficult because the cats become trap shy. In Australia, just treating annually for ectoparasites and worms in stray cats would cost about AU\$157 (approximately US $\$ 108$ ) per cat and would require monthly captures (Crawford et al. 2019). Because of the difficulty of catching cats more than once, most studies use visual assessments of TNR cats to determine the health of the cats. Some studies estimate that $21 \%$ of cats in a colony are blind or have scars or skin problems (Castro-Prieto and Andrade-Nunez 2018). Others have estimated that a large majority of the cats appear healthy ( $80 \%$ of cats in Auckland, New Zealand; Zito et al. 2019). However, visual assessments are likely to be inaccurate. Cats may appear to be healthy but have underlying conditions such as parasites and mastitis.

Finally, a typical treatment of a spayed or neutered cat is for a vet to recommend an e-collar to be placed on the cat to prevent them from opening/licking their incisions (Christy Layton, DVM, personal communication). There are no studies of the health outcomes of cats spayed or neutered after being released back outside without an e-collar.

\section{Do TNR programs increase the risk of transmitting diseases to humans or wildlife?}

Cats are host to a range of zoonotic diseases, such as rabies, toxoplasmosis, hookworms, and roundworms. The most common route of disease exposure to humans from feral cats is directly from bites and scratches. In Florida, cats are the most common domestic animal to expose humans to rabies, and on average 10 Floridians are exposed to rabies from feral cats each year (Florida Morbidity 
and Mortality Report 2017). In 2010, 303 rabid cats were reported through a national surveillance program in the USA (Blanton et al. 2011). Rabies is a lethal disease to humans, and exposure from feral cats requires aggressive and expensive postexposure treatment of the person exposed. In addition to rabies, feral cats may also be a reservoir for the flu virus. Laboratory studies have shown that cats can contract avian flu (H5N1) and could possibly transmit this virus to humans (Rimmelzwaan et al. 2006).

Cats also harbor ectoparasites, including fleas, ticks, and mites. Cat fleas carry numerous diseases that can impact people, including cat scratch fever (caused by the bacteria Bartonella henselae), flea-borne typhus (caused by several species of the bacteria Rickettsia spp.), and plague (caused by the bacteria Yersinia pestis; Gerhod and Jessop 2013). Cat scratch fever is a frequently diagnosed disease in children and young adults that have had contact with cats (McElroy et al. 2010).

Because feral cats deposit a large amount of urine and feces into the environment, contaminated soil around feral cat colonies is also a source of disease. Roundworm eggs and hookworm larvae reside in the soil and are transmitted to humans through skin contact with contaminated soil. For example, in Prague, in urban areas with feral cats, $45 \%$ of soil samples contained roundworm eggs. In California, it is estimated that 2,309 free-roaming cats contributed about 108 tons of feces into the landscape (Dabritz 2006). These contaminants can impact nearby waterbodies, such as wetlands, streams, and lakes. When it rains, feces and urine can be carried to nearby waters and pollute these areas. In Monterey Bay, California, the prevalence of Toxoplasma gondii infections in sea otters (Toxoplasma gondii is the causative agent of toxoplasmosis) has been attributed to coastal runoff of infected cat feces and urine into the ocean (Miller et al. 2002). In another example, feline leukemia virus (FeLV), which historically has not infected wild populations of panthers, caused the deaths of at least five endangered Florida panthers (Puma concolor coryi) between 2002 and 2004, and infections have been diagnosed in six additional panthers since 2010. These infections are believed to have been transferred to the panthers from domestic cats (https://myfwc.com/wildlifehabitats/wildlife/ panther/health/; accessed 1/7/20).

Trap-Neuter-Release cat colonies can be reservoirs for human diseases for several reasons. First, TNR colonies have high rates of immigration because they attract other cats into the colonies (Gunther et al. 2011). Often these newcomers are animals that do not have vaccinations and are susceptible to disease. As the number of susceptible animals increases, so does the risk of disease transmission (Gerhold and Jessup 2013). In addition, when people leave food for feral cat colonies, the food attracts local wildlife like raccoons, rodents, and opossums (Hernandez 2018) that can also transmit diseases to cats in the colonies, which increases the routes and frequency of disease transmission (Gerhold and Jessup 2013). Although some animals that have been spayed or neutered may also have been vaccinated against rabies, they are unlikely to have been vaccinated for other zoonotic diseases or treated for ectoparasites like fleas and ticks. Further, with rabies vaccinations, National Association of State Public Health Veterinarians have guidelines that state an animal needs an initial shot, a booster after one year, and then boosters every 1-3 years, depending on vaccine manufacturer recommendations (National Association of State Public Health Veterinarians, 2016). While feral cats that are returned to TNR colonies have been vaccinated for rabies, they are unlikely (if trap shy) to get the necessary booster shots, which means that these cats do not have life long immunity to rabies.

Feral cat colonies have been reported to control rats in urban areas (Glass et al. 2009), but this statement is questionable. In one study, cats in an alley with rats did reduce the population of rats by more than $50 \%$ in one year, but the following year, the number of rats increased by more than $100 \%$, despite the number of cats staying constant in the area (Glass et al. 2009). Further, cats may prey on rats, but that does not mean they stop preying on other animals in the area (Bradshaw 2006). Switching prey occurs depending on the abundance of certain prey species; so when rat populations go down, feral cats in the area will target other animal species, such as birds, frogs, etc.

Finally, feral cats are typically not socialized and can be a danger to people and pets nearby. Many feral cats can be aggressive and will bite and scratch people, and some become a public nuisance. For example, in Israel, 3,354 complaints were registered across five city councils about aggressive cats (Gunther et al. 2015). In 2018, more than 2000 cats were tested for rabies because of possible human exposure due to bites and scratches (Zito et al. 2019). In addition, stray cats enter yards and defecate and urinate in these yards, increasing the possibility of disease transmission to residents (Crawford et al. 2018). Thus, TNR colonies could have high rates of disease prevalence with an increased potential for human exposure. 


\section{Summary}

The above review of the TNR studies indicates that population outcomes cannot be predicted simply by the application of TNR: sometimes populations decline, and sometimes they do not. The lack of scientifically applied monitoring in studies makes reliable estimates of population sizes and TNR outcomes unreliable. What seems clear is that TNR colonies have high immigration rates via human-assisted or natural immigration, which keeps colony sizes from decreasing. In order to observe a decrease in population size in TNR colonies, high adoption/removal rates are needed to remove cats from the colony.

In theory, sterilizing enough cats so that the birth rate is less than the death rate would reduce the cat population in a given area. However, this assumes a closed population, a phenomenon that has not been observed in any of the studies. Instead, the studies observed cats immigrating into colonies, dispersing from other areas or being released into colonies by people. Even in a closed population, a large proportion of the colony must be sterilized (71\% to 94\%) before the population will decline over time (Andersen et al. 2004), which requires a significant input of resources. Another concern is whether TNR is humane and truly beneficial for the welfare of cats. As cited above, cats usually experience a painful death and suffer from various diseases and injuries during their lifetimes. Additionally, feral cat colonies are a source of public and wildlife health risk.

\section{Commentary by Authors}

Based on the science that we reviewed above, we offer commentary about the strategy of TNR to decrease feral cat populations, and we provide future directions to address the problem of homeless cats. Overall, we feel that TNR does not appear to decrease population size unless significant resources and efforts are made to remove animals via adoption. The animals that remain appear to live shorter lives and are subject to disease and injury. In our opinion, it is much more humane to capture a healthy cat, and, if cannot be adopted, have it euthanized instead of returning it to the outdoors, where it will suffer during its life.

The danger we see in TNR programs is that TNR may be seen as a viable solution to reducing feral cat populations, and that therefore less money and effort will go towards prevention of free-ranging cats. The practice of TNR and the establishment of TNR colonies is neither humane nor proven to be effective at reducing feral cat populations. Our review concurs with another published review that found that TNR colonies do not decrease without high adoption/ removal rates, and that these colonies are both a danger to the cats themselves and to nearby humans and wildlife (Longcore et al. 2009). We understand there is no easy solution to the feral cat population problem, but we (the authors) hope more funding is directed towards prevention. Keeping cats indoors and sterilizing them is not cruel and will help to keep these cats safe from disease or injury, allowing them to live longer (see American Bird Conservancy Cats Indoors Campaign). People should not feed outdoor cats but should rather help them to be adopted. People who care about the welfare of cats can support funding for local animal shelters and can increase public education for the adoption of cats. Euthanizing sick, injured, or unadoptable cats may be the only solution in cases where adoption rates are low. We maintain, based on the best available science, that TNR is not a viable solution in most situations. Overall, we view TNR strategies as inhumane to the cats themselves and potentially dangerous to humans, pets, and wildlife.

\section{Literature Cited}

Andersen, M. C., B. J. Martin, and G. W. Roemer. 2004. "Use of Matrix Population Models to Estimate the Efficacy of Euthanasia Versus Trap-Neuter-Return for Management of Free-Roaming Cats." Journal of the American Veterinary Medical Association 225: 1871-1876.

Blanton, J. D., D. Palmer, J. Dyer, and C. E. Rupprecht. 2011. "Rabies Surveillance in the United States during 2010." J. Am. Vet. Med. Assoc. 239: 773-783.

Boone, J. D., P. S. Miller, J. R. Briggs, V. A. Benka, D. F. Lawler, M. Slater, J. K. Levy, and S. Zawistowski. 2019. “A Long-Term Lens: Cumulative Impacts of Free-Roaming Cat Management Strategy and Intensity on Preventable Cat Mortalities." Front. Vet. Sci. https://doi.org/10.3389/ fvets.2019.00238

Bradshaw, J. W. S. 2006. "The Evolutionary Basis for the Feeding Behavior of Domestic Dogs (Canis familiaris) and Cats (Felis catus)." J. Nutr. 136: 1927S-1931S.

Castro-Prieto, J., and M. J. Andrade-Nunez. 2018. "Health and Ecological Aspects of Stray Cats in Old San Juan, Puerto Rico: Baseline Information to Develop an Effective Control Program." P. R. Health Sci. J.37: 110-114.

Castillo, D., and A. L. Clarke. 2003. “Trap/Neuter/Release Methods Ineffective in Controlling Domestic Cat 'Colonies' on Public Lands.' Natural Areas Journal 23(3): 247-253. 
Centonze, L. A., and J. K. Levy. 2002. "Characteristics of Free-Roaming Cats and their Caretakers." J. Am. Vet. Med. Assoc. 220: 1627-1633.

Chalkowski, K., A. E. Wilson, C. A. Lepczyk, and S. Zohdy. 2019. "Who Let the Cats Out? A Global Meta-Analysis on Risk of Parasitic Infection in Indoor Versus Outdoor Domestic Cats (Felis catus)." Biol. Lett. 15(4): 20180840.

Crawford, H. M., M. C. Calver, and P. A. Fleming. 2019. "A Case of Letting the Cat Out of The Bag-Why Trap-NeuterReturn Is Not an Ethical Solution for Stray Cat (Felis catus) Management." Animals 9(4): Article 171.

Crawford, H. M., J. B. Fontaine, M. C. Calver. 2018. “Ultrasonic Deterrents Reduce Nuisance Cat (Felis catus) Activity on Suburban Properties." Glob. Ecol. Conserv. 15: e00444, doi:10.1016/j.gecco.2018.e00444.

Dabritz, H. A., R. Atwill, I. A. Gardner, M. A. Miller, and P. A. Conrad. 2006. "Outdoor Fecal Deposition by FreeRoaming Cats and Attitudes of Cat Owners and Nonowners toward Stray Pets, Wildlife, and Water Pollution." J. Am. Vet. Med. Assoc. 229: 74-81 doi:10.2460/javma.229.1.74.

Foley, P., J. E. Foley, J. K. Levy, and T. Paik. 2005. "Analysis of the Impact of Trap-Neuter-Return Programs on Populations of Feral Cats." JAVMA Vol 227 No. 11: 1775-1781.

Gerhold, R. W., and D. A. Jessup. 2013. "Zoonotic Diseases Associated with Free-Roaming Cats." Zoonoses and Public Health 60(3): 189-195.

Glass, G. E., L. C. Gardner-Santana, R. D. Holt, J. Chen, T. M. Shields, M. Roy, S. Schachterle, and S. L. Klein. 2009. "Trophic Garnishes: Cat-Rat Interactions in an Urban Environment." PLoS ONE 4: e5794. doi:10.1371/journal. pone.0005794.

Gunther, I., T. Raz, O. Berke, and E. Klement. 2015. "Nuisances and Welfare of Free-Roaming Cats in Urban Settings and Their Association with Cat Reproduction." Prev. Vet. Med. 119: 203-210. doi:10.1016/j.prevetmed.2015.02.012.

Gunther, I., H. Finkler, and J. Terkel. 2011. "Demographic Differences between Urban Feeding Groups of Neutered and Sexually Intact Free-Roaming Casts Following a Trap-Neuter-Return Procedure." J. Am. Vet. Med. Assoc. 238: $1134-1140$.
Hatley, P. J. 2003. "Feral Cat Colonies in Florida: The Fur and Feathers Are Flying." Journal of Land Use 18: 441-465.

Hernandez, S. M., K. A. T. Loyd, A. N. Newton, M. Gallagher, B. L. Carswell, and K. J. Abernathy. 2018. "Activity Patterns and Interspecific Interactions of Free-Roaming, Domestic Cats in Managed Trap-Neuter-Return Colonies." Appl. Anim. Behav. Sci. 202: 63-68.

Ireland, T., and R. M. Neilan. 2016. "A Spatial Agent-Based Model of Feral Cats and Analysis of Population and Nuisance Controls." Ecological Modelling 337: 123-136.

Jessup, D. 2004. "The Welfare of Feral Cats and Wildlife." Journal of the American Veterinary Medical Association 225(9):1377-83

Kreisler, R. E., H. N. Cornell, and J. K. Levy. 2019. “Decrease in Population and Increase in Welfare of Community Cats in a Twenty-Three Year Trap-Neuter-Return Program in Key Largo, FL: The ORCAT Program." Front. Vet. Sci. 6 : 7.

Lefkaditis, M. A., A. V. Sossidou, A. H. Panorias, S. E. Koukeri, A. I. Paştiu, and L. V. Athanasiou. 2015. "Urban Stray Cats Infested by Ectoparasites with Zoonotic Potential in Greece." Parasitol. Res. 114: 3931-3934. doi:10.1007/ s00436-015-4688-4.

Levy, J. K., and P. C. Crawford. 2004. "Humane Strategies for Controlling Feral Cat Populations." J. Am. Vet. Med. Assoc. 9: 1354-1360.

Levy, J. K., D. W. Gale, L. A. Gale. 2003. "Evaluation of the Effect of a Long-Term Trap-Neuter-Return and Adoption Program on a Free-Roaming Cat Population." J. Am. Vet. Med. Assoc. 222: 42-46.

Levy, J. K., N. M. Isaza, and K. C. Scott. 2014. "Effect of High-Impact Targeted Trap-Neuter-Return and Adoption of Community Cats on Cat Intake to a Shelter." Vet J. 201:269-74. doi: 10.1016/j.tvjl.2014.05.001

Longcore, T., C. Rich, and L. M. Sullivan. 2009. "Critical Assessment of Claims Regarding Management of Feral Cats by Trap-Neuter-Return." Conservation Biology 23(4): 887-894.

Loss, S. R., T. Will, T. Longcore, and P. P. Marra. 2018. "Responding to Misinformation and Criticisms Regarding United States Cat Predation Estimates." Biological Invasions 20: 3385-3396. 
Ma, X., B. P. Monroe, J. M. Cleaton, L. A. Orciari, C. M. Gigante, J. D. Kirby, R. B. Chipman, C. Fehlner-Gardier, V. Gutierrez Cedillo, B. W. Petersen, V. Olson, and R. M. Wallace. 2020. "Rabies Surveillance in the United States during 2018." Journal of the American Veterinary Medical Association. 256: 195-208.

McElroy, K. M., B. L. Blagburn, E. B. Breitschwerdt, P. S.Mead, and J. H. McQuiston. 2010. "Flea-Associated Zoonotic Diseases of Cats in the USA: Bartonellosis, Flea-Borne Rickettsioses, and Plague." Trends Parasitol. 26: 197-204.

Miller, M. A., I. A. Gardner, C. Kreuder, D. M. Paradies, K. R. Worcester, D. A. Jessup, E. Dodd, M. Harris, J. A. Ames, A. E. Packham, and P. A. Conrad. 2002. "Coastal Freshwater Runoff Is a Risk Factor for Toxoplasma gondii Infection of Southern Sea Otters (Enhydra lutris nereis)." International journal for parasitology. 32(8): 997-1006.

Mohd Zain, S. N., N. Sahimin, P. Pal, and J. W. Lewis. 2013. "Macroparasite Communities in Stray Cat Populations from Urban Cities in Peninsular Malaysia." Vet. Parasitol. 196: 469-477. doi:10.1016/j.vetpar.2013.03.030.

National Association of State Public Health Veterinarians. 2016. "Compendium of Animal Rabies Prevention and Control." JAVMA 248(5): 505-517.

Natoli, E., L. Maragliano, G. Cariola, A. Faini, R. Bonanni, S. Cafazzo, and C. Fantini. 2006. "Management of Feral Domestic Cats in the Urban Environment of Rome (Italy)." Prev Vet Med. 77(3-4):180-5.

Nutter, F. B., J. F. Levine, and M. K. Stoskopf. 2004. "Reproductive Capacity of Free-Roaming Domestic Cats and Kitten Survival Rate." J. Am. Vet. Med. Assoc. 225(9): 1399-1402.

Nutter, F. B. 2006. Evaluation of a Trap-Neuter-Return Management Program for Feral Cat Colonies: Population Dynamics, Home Ranges, and Potentially Zoonotic Diseases. North Carolina State University, Ann Arbor, MI, USA

Rimmelzwaan, G. F., D. van Riel, M. Baars, T. M. Bestebroer, G. van Amerongen, R. A. Fouchier, A. D. Osterhaus, and T. Kuiken. 2006. "Influenza A Virus (H5N1) Infection in Cats Causes Systemic Disease with Potential Novel Routes of Virus Spread within and between Hosts." Am. J. Pathol. 168: 176-183. doi:10.2353/ajpath.2006.050466.
Schmidt, P. M., R. R. Lopez, and B. A. Collier. 2007. "Survival, Fecundity, and Movements of Free-Roaming Cats." J. of Wildlife Management 71: 915-919.

Spehar, D. D., and P. J. Wolf. 2017. "An Examination of an Iconic Trap-Neuter-Return Program: The Newburyport, Massachusetts Case Study." Animals 7(11): 81.

Spehar, D. D., and P. J. Wolf. 2018. "A Case Study in Citizen Science: The Effectiveness of a Trap-Neuter-Return Program in a Chicago Neighborhood." Animals 8(1): 14.

Swarbrick, H., and J. Rand. 2018. "Application of a Protocol Based on Trap-Neuter-Return (TNR) to Manage Unowned Urban Cats on an Australian University Campus." Animals 8(5): 77.

Vnuk, D., H. Capak, V. Gusak, D. Maticic, M. Popovic, and N. B. Bottegaro. 2016. "Metal Projectile Injuries in Cats: Review of 65 Cases (2012-2014)." J. Feline Med. Surg. 18: 626-631. doi:10.1177/1098612X15590869.

Waap, H., J. Gomes, and T. Nunes. 2014. "Parasite Communities in Stray Cat Populations from Lisbon, Portugal." J. Helminthol. 88: 389-395. doi:10.1017/S0022149X1300031X.

Warner, R. 1985. "Demography and Movements of Free-Ranging Domestic Cats in Rural Illinois." J Wildlife Management. 49: 340-346.

Wilson, P. M., C. R. Tidemann, and H. R. C. Meischke. 1994. “Are Cats on Rubbish Dumps a Problem?" In: Proceedings of the Urban Animal Management, Sydney, Australia. 163-174.

Zito, S., J. M. Walker, C. Gates, and D. Arnja. 2019. "A Preliminary Description of Companion Cat, Managed Stray Cat, and Unmanaged Stray Cat Welfare in Auckland, New Zealand Using a 5-Component Assessment Scale." Front. Vet. Sci. 21. https://doi.org/10.3389/fvets.2019.00040 